\title{
Unifying energy harvesting, sensing, and communication for ultra-low power structure health monitoring
}

\author{
Guoqing Zhou ${ }^{\text {** }}$ and Jingxian $\mathrm{Wu}^{2}$
}

\begin{abstract}
A new ultra-low power (ULP) wireless sensor network (WSN) is proposed to monitor the vibration properties of critical structures such as buildings, bridges, and the wings and bodies of aircrafts. The new scheme integrates energy harvesting, data sensing, and wireless communication into a unified process, and it is fundamentally different from all the existing WSNs. In the new WSN, self-powered sensors are employed to harvest vibration energy and measure vibration intensity simultaneously, by utilizing the fact that the harvested energy accumulated through time is proportional to the vibration amplitude and frequency. Once the harvested energy reaches a threshold, it is released as an impulse with a wireless transmitter. An estimate of the structure vibration intensity can then be obtained by measuring the number of binary impulses in a unit time. Such an approach does not require complicated analog-to-digital conversion or signal processing, and it can achieve an ULP performance unrivaled by existing technologies. Optimum and sub-optimum impulse density estimation algorithms are proposed to take advantage of the spatial correlation among signals from the sensors. Analytical and simulation results demonstrate that the proposed scheme can efficiently operate at a low signal-to-noise ratio (SNR).
\end{abstract}

Keywords: Wireless sensor network; Structure health monitoring; Energy harvesting; Maximum a posteriori (MAP) detection

\section{Introduction}

Wireless sensor network (WSN) designed for structure health monitoring (SHM) is expected to operate uninterrupted over a long period of time, under the constraints of extremely limited battery capacity or very small energyscavenging devices. Ultra-low power (ULP) consumption is one of the most formidable challenges faced by the development of WSN for the autonomous monitoring of critical structures, such as bridges, buildings, [1], and aircrafts and spacecrafts [2]. Hence, an extremely stringent power budget is required to power the operation of a wireless sensor, which transmits the measured data to a fusion center (FC) through a wireless link.

Recently, there have been considerable efforts devoted to the development of WSN for SHM systems [1-6]. Most of the sensing systems are built with

*Correspondence: guoqing.zhou@litepoint.com

${ }^{1}$ Litepoint Corporation, 965 West Maude Avenue, Sunnyvale, CA 94085, USA

Full list of author information is available at the end of the article commercial-off-the-shelf (COTS) wireless sensor nodes, such as Mica-Z Mote [3,4], Mica-2 Mote [5], and iMote [6], etc. Even though these modules are designed with low-power consumption as one of the design objectives, their structures still follow a conventional sensing framework, which includes sensing, analog-to-digital conversion (ADC), digital signal processing (DSP), and wireless transmission. These modules are designed separately, and they do not directly take advantage of the unique features of SHM systems. In order to achieve ULP performance, we need to break free from the conventional sensing frameworks and seek fundamentally new WSN structures. SHM possesses many unique features that can be exploited to facilitate the ULP design. Many of the structures, such as bridges, have a very slow changing rate, e.g., new data might only need to be collected once every few seconds or minutes. As a result, SHM has long latency tolerance with ultra-low data rate. In addition, Data collected by spatially distributed sensors often

\section{Springer}

(c) 2015 Zhou and Wu; licensee Springer. This is an Open Access article distributed under the terms of the Creative Commons Attribution License (http://creativecommons.org/licenses/by/4.0), which permits unrestricted use, distribution, and reproduction in any medium, provided the original work is properly credited. 
contain redundancy $[7,8]$, which can be used to achieve better power efficiency.

In this paper, we propose a new type of battery-free ULP WSN by integrating energy harvesting, data sensing, and wireless communication into a unified process. The system is designed to monitor the structure vibration intensity, such as vibration amplitude and frequency, which provides useful information about the local stress intensity and the dynamic behaviors of the structure [9]. Vibration generates energy that can be harvested by a sensor with piezoelectric devices $[10,11]$. The harvested energy is expected to power the operations of the entire sensor node. However, due to the low efficiency of current piezoelectric materials, the harvested energy level is usually much lower compared to that required to perform any regular sensing, ADC, DSP, or communication functions. Therefore, conventional sensing or communication techniques can no longer be applied in such a system.

We propose to address this problem by utilizing the correlation between energy and vibration, i.e., the harvested energy accumulated through time is proportional to the local vibration amplitude and frequency. Once the harvested energy reaches a predefined threshold, the energy is released in the form of an impulse. The receiver can then obtain an estimate of the vibration intensity by observing the impulse density, i.e., the number of impulses in unit time. Such an integrated harvesting, sensing, and communication (IHSC) process exploits the unique features of SHM systems, and it is fundamentally different from conventional sensing schemes.

The impulses from the sensors are detected at the FC through an optimum multi-node maximum a posteriori (MAP) detector, which exploits the spatial correlation among the signals from the sensors. It should be noted that the proposed multi-node MAP detector needs to detect the presence of impulses from different sensors, and this is different from the decentralized detection in the literature [12-16], where the FC only needs to detect the presence of a single event by collecting the noisefree or noisy local detections from a number of sensors. The multi-node MAP detector requires the a priori probabilities of the impulses, which are not readily available at the receiver. We propose an iterative method to estimate the a priori probability of the impulses at the FC. Simulations show that the iterative method usually converges in less than five iterations. The theoretical mean square error (MSE) of the estimated impulse density is derived for a system operating in a Rayleigh fading channel. Both analytical and simulation results show that the proposed IHSC scheme can operate at a very low signalto-noise ratio (SNR) by effectively utilizing the spatial signal correlation.

The remainder of this paper is organized as follows. A new WSN structure with integrated harvesting, sensing and communication is presented in Section 2. The optimum impulse density estimation algorithms and the corresponding theoretical analysis are proposed in Section 3. Simulation results are given in Section 4, and Section 5 concludes the paper.

\section{A new WSN structure with integrated harvesting, sensing, and communication}

Consider a WSN consisting of a large number of lowcost battery-free wireless sensor nodes uniformly distributed over the monitored structure. As illustrated in Figure 1, each sensor node is equipped with a selfpowered nanowire sensor [10] for energy harvesting and data sensing, and a simple radio frequency (RF) transmitter, such as a simple resistor-capacitor (RC) oscillator. The sensor performs the IHSC operation described as follows.

Definition 1. (IHSC): The energy collected by the nanowire piezoelectric sensor is used to charge a capacitor. Once the harvested energy reaches a predefined threshold, $E_{\mathrm{TH}}$, the energy is released as a single impulse through the RF transmitter. Then the receiver can obtain an estimate of the structure vibration intensity by measuring the impulse density.

In the above IHSC procedure, it is assumed that the energy harvesting rate, i.e., the energy harvested in unit time, is proportional to the structure vibration intensity, i.e., vibration amplitude and frequency. As a result, the amount of time required for the harvested energy to reach $E_{\mathrm{TH}}$ is inversely proportional to the vibration intensity. Therefore, the structure vibration information is carried in the form of the time delay between two consecutive impulses, or the number of impulses in unit time. The proposed IHSC scheme utilizes the correlation among structure vibration, energy, and time to get an estimate of the structure vibration intensity.

Given the fact that the structure vibration is highly correlated across the spatial domain, the density information collected by spatially distributed sensors is correlated. Such correlation information can be exploited by the FC to increase the estimation accuracy even at an extremely low SNR. Optimum and sub-optimum impulse density estimation algorithms will be developed in the next section to exploit the spatial correlation among sensors.

To facilitate analysis, we have the following assumptions regarding the statistical properties of the structure vibration.

A.1) The amount of time for the harvested energy to reach $E_{\mathrm{TH}}$ is an exponentially distributed random variable (RV) with mean $\mu$. A higher vibration intensity yields a smaller $\mu$.

A.2) The time is discretized into small intervals with duration $T_{s} \ll \mu$. For each interval, the receiver 


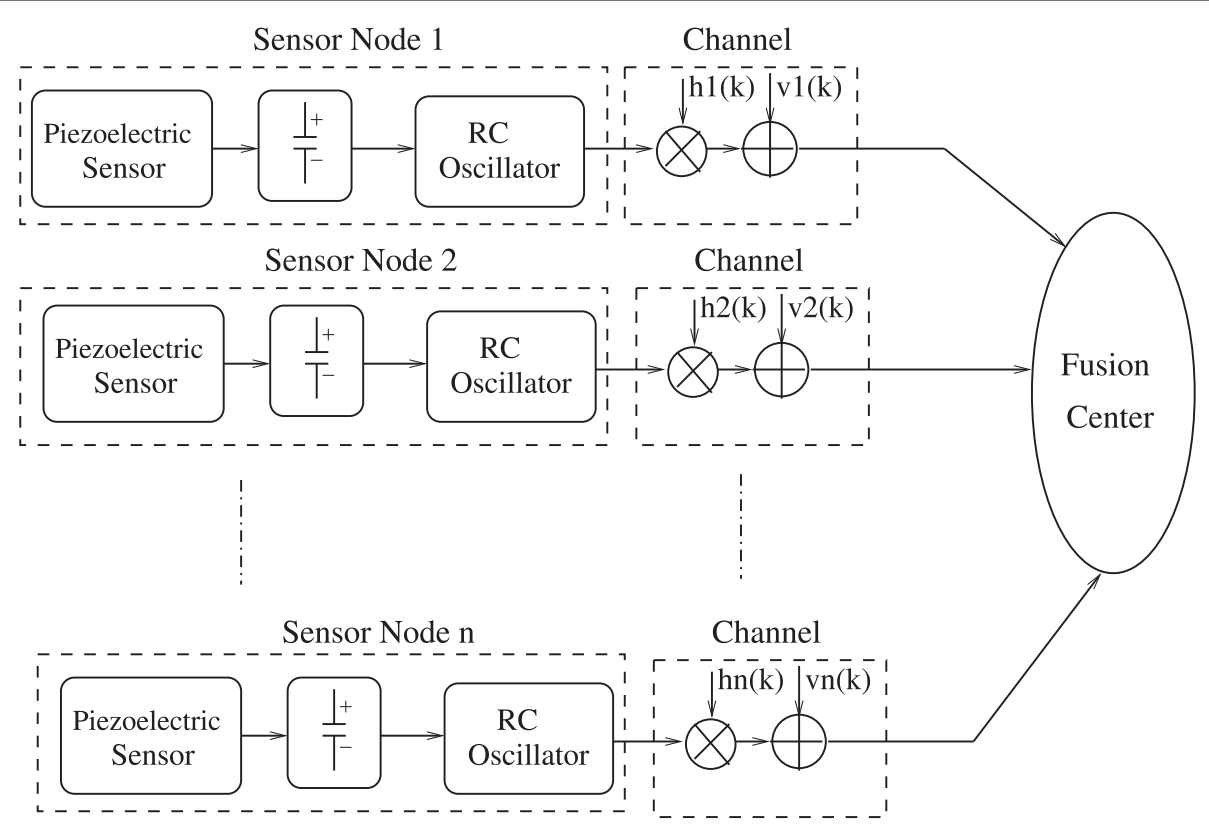

Figure 1 The block diagram of the wireless sensor network structure with integrated harvesting, sensing, and communication

performs detection to find whether there is an impulse in the interval. Define a RV, $x_{n k}$, where $x_{n k}=1$ represents an impulse is transmitted by the node $n$ at the $k$ th detection interval and 0 otherwise. Based on Assumption A.1), it can be easily shown that $x_{n k}$ is a Bernouli RV with the parameter

$$
p=P\left(x_{n k}=1\right)=1-e^{-\frac{T_{s}}{\mu}} .
$$

A.3) Data collected from different sensor nodes are correlated. The vibration correlation is translated to the correlation among the Bernouli RVs, $\left\{x_{n k}\right\}_{n=1}^{N}$. The normalized covariance coefficient between $x_{m k}$ and $x_{n k}$ is

$$
\phi_{m n} \triangleq \frac{\mathbb{E}\left\{\left[x_{m k}-\bar{x}_{m k}\right]\left[x_{n k}-\bar{x}_{n k}\right]\right\}}{\sqrt{\sigma_{m}^{2} \sigma_{n}^{2}}}=\theta^{|m-n|},
$$

where $\theta \in[0,1]$ is the spatial correlation coefficient, $\bar{x}_{m k}$ is the mean of $x_{m k}, \sigma_{m}^{2}$ is the variance of $x_{m k}$ and $\mathbb{E}(\cdot)$ is the expectation operator.

A.4) Sensors deliver the impulses to the FC through an orthogonal media access control (MAC) scheme, such as the frequency division multiplexing access (FDMA), to achieve a collision-free communication the FC.

With the above assumptions, the signal received by the FC from the $n$th sensor at the $k$ th interval can be represented as

$$
y_{n k}=\sqrt{E_{\mathrm{TH}}} \cdot h_{n k} \cdot x_{n k}+v_{n k}
$$

where $\sqrt{E_{\mathrm{TH}}}$ is the amplitude of the transmitted signal, $h_{n k}$ is the gain of the channel, and $v_{n k}$ is the additive white Gaussian noise (AWGN) with double-sided power spectral density $N_{0} / 2$.

Based on the model in (3), define the average impulse density of the $n$th sensor node over a duration of $K T_{s}$ as

$$
V_{n}=\frac{\sum_{k=1}^{K} x_{n k}}{K T_{s}}
$$

With the proposed IHSC scheme, the impulse density is proportional to the vibration intensity of the monitored structure, thus it can be used as an important indicator of the health condition of the structure.

\section{Optimum impulse density estimation}

In this section, we present an optimum receiver for the estimation of the impulse density, $V_{n}$, in a multi-node system employing the IHSC scheme.

\subsection{Iterative impulse density estimation}

To utilize the spatial data correlation, we will jointly estimate the data from all the nodes, $\mathbf{x}_{k}=\left[x_{1 k}, \cdots, x_{n k}\right]^{T} \in$ $\mathcal{B}^{N \times 1}$, based on the received signal vector, $\mathbf{y}_{k}=$ $\left[y_{1 k}, \cdots, y_{N k}\right]^{T} \in \mathcal{C}^{N \times 1}$, where $(\cdot)^{T}$ represents matrix transpose, and $\mathcal{C}$ is the set of complex numbers. At the detection interval $k$, the multi-node MAP detection of $\mathbf{x}_{k}$ is

$$
\hat{\mathbf{x}}_{k}=\underset{\mathbf{b} \in \mathcal{B}^{N}}{\operatorname{argmax}} p\left(\mathbf{y}_{k} \mid \mathbf{x}_{k}=\mathbf{b}\right) P\left(\mathbf{x}_{k}=\mathbf{b}\right),
$$

where $p\left(\mathbf{y}_{k} \mid \mathbf{x}_{k}=\mathbf{b}\right)$ takes the form of a multi-variant Gaussian probability density function (pdf) with the mean 
vector $\mathbf{b}$ and the covariance matrix $N_{0} \mathbf{I}_{N}$, with $\mathbf{I}_{N}$ being a size- $N$ identity matrix,

$$
p\left(\mathbf{y}_{k} \mid \mathbf{x}_{k}=\mathbf{b}\right)=\frac{1}{\left(\pi N_{0}\right)^{N}} \exp \left\{-\frac{1}{N_{0}} \sum_{n=1}^{N}\left|y_{n k}-\sqrt{E_{\mathrm{TH}}} \cdot h_{n k} \cdot b_{n}\right|^{2}\right\} .
$$

It should be noted that $\mathbf{x}_{k}$ are mutually correlated with the normalized correlation coefficient defined in (2).

The MAP detection rule described in (5) requires the knowledge of $p_{\mathbf{b}} \triangleq P\left(\mathbf{x}_{k}=\mathbf{b}\right)$, which is unknown at the receiver. To solve this problem, we propose to perform joint estimation of $p_{\mathbf{b}}$ and $\mathbf{x}_{k}$ with an iterative method.

At the beginning of the iteration, it is assumed that the data from all the nodes are uncorrelated, and the initial value of the a priori probability is $p_{\mathbf{b}}^{(0)}=0.5^{N}$. During the $i$ th iteration, we apply $p_{\mathbf{b}}^{(i-1)}$ from the $(i-1)$ th iteration to (5) and get the estimates $\hat{\mathbf{x}}_{k}^{(i)}$, for $k=1, \cdots, K$. The estimated values are then used to obtain an estimate of $p_{\mathbf{b}}$ as

$$
p_{\mathbf{b}}^{(i)}=\frac{1}{K} \sum_{k=1}^{K} \delta\left(\hat{\mathbf{x}}_{k}^{(i)}-\mathbf{b}\right), \quad \forall \mathbf{b} \in \mathcal{B}^{N \times 1}
$$

where the indicator function $\delta(\mathbf{0})=1$, and $\delta(\mathbf{x})=0$ if $\mathbf{x} \neq \mathbf{0}$. It should be noted that the estimation of the $a$ priori probability in (7) implicitly takes into consideration of the mutual correlation among the data in $\mathbf{x}_{k}$.

The iteration will be terminated if $\max _{\mathbf{b}}\left\{p_{\mathbf{b}}^{(i)}-p_{\mathbf{b}}^{(i-1)}\right\}<\epsilon$, or the number of iterations exceeds a predefined threshold. At the end of the iteration, we can get an estimate of the impulse density of the $n$th node as

$$
\hat{V}_{n}=\frac{1}{K T_{s}} \sum_{k=1}^{K} \hat{x}_{n k} .
$$

Simulation results demonstrate that the proposed iteration method usually converges after less than five iterations.

The optimum MAP detection requires the exhaustive search of the space $\mathcal{B}^{N}$, and the complexity grows exponentially with the node number, $N$. In a practical environment, the correlation between two nodes decreases as their distance increases. Therefore, joint detection of two nodes that are further apart would render very small performance gains over the case that they are detected separately.

In recognition of this fact, when $N$ is large, we propose to divide the $N$ nodes into $G$ groups. Each group contains up to $N_{g}=\left\lceil\frac{N}{G}\right\rceil$ adjacent nodes. The iterative MAP algorithm can then be applied to each group separately. Such a method features a tradeoff between complexity and performance. The optimum performance is obtained by setting $N_{g}=N$ with the highest complexity. The complexity can be reduced by decreasing $N_{g}$, at the cost of slightly decreased performance. Our simulation results show that the performance at $N_{g}=4$ is very similar to its optimum counterpart for a wide range of correlation coefficient.

\subsection{Performance analysis}

The MSE of the estimated impulse density in a multi-node system with correlated information is presented in this section.

To facilitate analysis, define $U_{n}=\sum_{k=1}^{K} x_{n k}$, and $\hat{U}_{n}=$ $\sum_{k=1}^{K} \hat{x}_{n k}$. Then both $U_{n}$ and $\hat{U}_{n}$ are binomial RVs, i.e., $U_{n} \sim B(K, p)$, and $\hat{U}_{n} \sim B(K, q(N, \theta))$, with $p=P\left(x_{n k}=\right.$ 1) and $q(N, \theta)=P\left(\hat{x}_{n k}=1 \mid N, \theta\right)$. Then the MSE can be written as $\sigma^{2}=\frac{1}{\left(K T_{s}\right)^{2}} \mathbb{E}\left(\left|U_{n}-\hat{U}_{n}\right|^{2}\right)$, and it can be calculated from the following proposition.

Proposition 1. For a multi-node system that employs the optimum multi-node MAP detection, the MSE of the estimated impulse density for each sensor node can be calculated by

$\sigma^{2}=\frac{1}{K T_{s}^{2}}\left\{(K-1)[p-q(N, \theta)]^{2}+p+q(N, \theta)-2 \alpha(N, \theta)\right\}$.

where $\alpha(N, \theta)=\mathbb{E}\left[x_{n k} \hat{x}_{n k} \mid N, \theta\right]$ is the cross-correlation between $x_{n k}$ and $\hat{x}_{n k}$.

Proof. The proof is in Appendix A.

The calculation of the MSE requires the knowledge of $q(N, \theta)$ and $\alpha(N, \theta)$. The analytical evaluations of the two parameters for arbitrary $N$ are quite tedious.

Here we only give the analytical expressions for $N=1$, i.e., the information from each node is detected independently, thus $q(1, \theta)=q(1)$ because the correlation $\theta$ is not used during the detection.

Lemma 1. The value of $q(1)=P\left(\hat{x}_{n k}=1 \mid N=1\right)$ in a Rayleigh fading channel is given as follows

$$
q(1)=p\left(1-P_{m}\right)+(1-p) P_{f},
$$

where

$$
\begin{aligned}
P_{m} & =P\left\{\hat{x}_{n k}=0 \mid x_{n k}=1\right\} \\
& =\int_{0}^{\infty} Q\left(\frac{E_{\mathrm{TH}} \cdot x-\eta_{10}}{\sqrt{2 N_{0} \cdot E_{\mathrm{TH}} \cdot x}}\right) \exp (-x) d x, \\
P_{f} & =P\left\{\hat{x}_{n k}=1 \mid x_{n k}=0\right\} \\
& =\int_{0}^{\infty} Q\left(\frac{E_{\mathrm{TH}} \cdot x+\eta_{10}}{\sqrt{2 N_{0} \cdot E_{\mathrm{TH}} \cdot x}}\right) \exp (-x) d x,
\end{aligned}
$$

are the probabilities of missing detection and false alarm, respectively, $Q(x)=\frac{1}{\sqrt{2 \pi}} \int_{x}^{\infty} \exp \left(-\frac{u^{2}}{2}\right) d u$ is the Gaussian-Q function, and $\eta_{10}=N_{0} \log \frac{1-p}{p}$. 


\section{Proof. The proof is in Appendix B}

The cross-correlation $\alpha(1)=\alpha(1, \theta)$ can be evaluated as $\alpha(1)=P\left(\hat{x}_{n k}=1, x_{n k}=1\right)=\left(1-P_{m}\right) p$.

The probability expressions of missing and false alarm given in Lemma 1 involve integrations over infinite limits, which might cause instability during numerical evaluations. We propose to calculate the results in Lemma 1 by using the Laguerre's method [17]

$$
\int_{0}^{\infty} f(a) \exp (-a) d a=\sum_{i=1}^{M} w_{i} f\left(a_{i}\right)+R_{M},
$$

where $a_{i}$ is the $i$ th zeros of the Lagueree polynomials $L_{I}(a)$, weights $w_{i}$ is defined as $w_{i}=\frac{a_{i}}{(M+1)^{2}\left[L_{I+1}\left(a_{i}\right)\right]^{2}}$, and $R_{M}$ is the remainder term.

Based on (12), $P_{m}$ and $P_{f}$ in a Rayleigh fading channel can be represented as,

$$
\begin{aligned}
P_{m} & \approx \sum_{i=1}^{M} w_{i} Q\left(\frac{E_{\mathrm{TH}} \cdot x_{i}-\eta_{10}}{\sqrt{2 N_{0} \cdot E_{\mathrm{TH}} \cdot x_{i}}}\right), \\
P_{f} & \approx \sum_{i=1}^{M} w_{i} Q\left(\frac{E_{\mathrm{TH}} \cdot x_{i}+\eta_{10}}{\sqrt{2 N_{0} \cdot E_{\mathrm{TH}} \cdot x_{i}}}\right) .
\end{aligned}
$$

When $N>1$, the value of $q(N, \theta)$ and $\alpha(N, \theta)$ can be evaluated through numerical simulations, the results of which can then be substituted into (9) to obtain the MSE.

\section{Simulation results}

Simulation results are presented in this section to verify the performance of the proposed ULP IHSC scheme and the optimum and sub-optimum impulse density estimation algorithms.

In the simulation, it is assumed that the mean, $\mu$, of the exponentially distributed energy harvesting time is 1 $\mathrm{s}$. The detection duration is $T_{s}=10 \mathrm{~ms}$. The correlated Bernoulli RVs, $x_{n k}$, are generated by using the method described in [18]. The iterative impulse density detection is performed over $100 \mathrm{~s}$, which corresponds to $K=10^{4}$ detection intervals. The average SNR is calculated as $v=$ $\frac{E_{\mathrm{TH}} T_{s}}{N_{0} \mu}$. Unless otherwise stated, the receiver does not have any $a$ priori knowledge of the probability, $P\left(\mathbf{x}_{k}\right)$, or spatial correlation coefficient, $\theta$.

Figure 2 shows the MSE of the estimated impulse density for a one-node and a two-node system with the optimum MAP detection at the FC. The simulation results obtained from systems with both known and unknown $a$ priori probability at the receiver are plotted in the figure for comparison. The spatial correlation coefficient of the two-node system is $\theta=0.9$. We have the following observations of the results. First, the system can operate at extremely low SNR due to the low duty cycle and the innovative IHSC scheme. When SNR $=0 \mathrm{~dB}$, an MSE of $2 \times 10^{-4}$ and $3 \times 10^{-3}$ is achieved by the one-node and

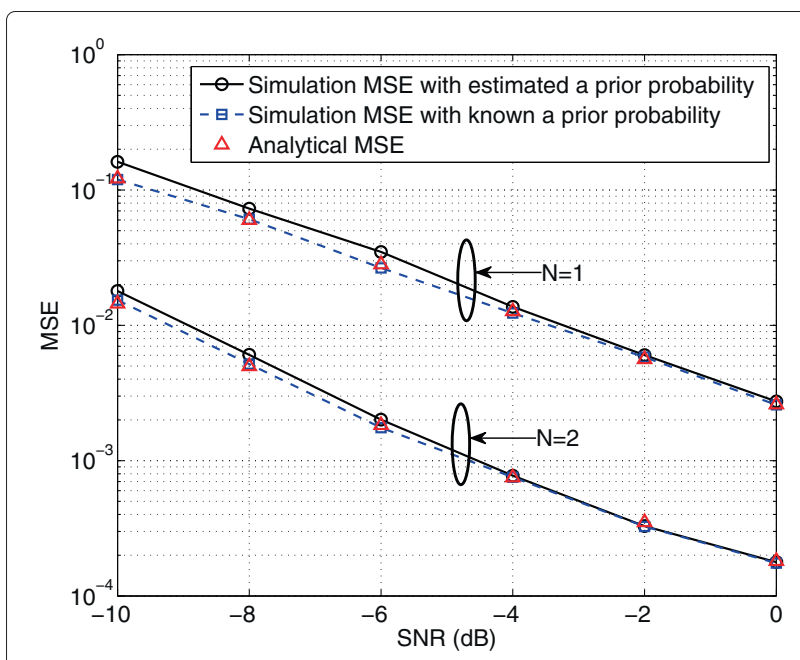

Figure 2 MSE for systems with optimum impulse density estimation.

two-node systems, respectively. Second, when SNR $>-4$ $\mathrm{dB}$, the iterative estimation methods with unknown $a$ priori probability can achieve a performance that is almost identical to that of a system with known a priori probability. This demonstrates the effectiveness of the proposed iterative estimation method. Third, the analytical results match very well with the simulation results when the $\mathrm{SNR}>-4 \mathrm{~dB}$. Fourth, at MSE $=10^{-2}$, the two-node system outperforms the one-node system by $5.5 \mathrm{~dB}$. The performance improvement is contributed by the utilization of the spatial node correlation.

The impact of the spatial correlation coefficient, $\theta$, on the MSE performance is shown in Figure 3 for a two-node system. As expected, the MSE performance improves consistently as $\theta$ increases. At MSE $=10^{-3}$, the system with $\theta=1$ outperforms that with $\theta=0.5$ by $6.3 \mathrm{~dB}$. The results demonstrate that the proposed algorithm can effectively utilize the spatial correlation between the nodes. Meanwhile, it shows that the stronger the spatial correlation, the better performance can be obtained.

In Figure 4, the MSE of estimated impulse density is shown for a multi-node system using MAP detection scheme with different group sizes at the FC. The SNR is $-10 \mathrm{~dB}$. As expected, the performance improves as the group size increases. Most of the performance gains are achieved when $N_{g}<4$, and they gradually diminish as $N_{g} \geq 4$, for all the systems considered in this example. Based on the results, a window size of 4 yields the best tradeoff between complexity and performance for a wide range of the correlation coefficient, $\theta$.

\section{Conclusions}

A new paradigm of an integrated harvesting, sensing, and communication scheme was proposed for ultra-low power structure health monitoring. The IHSC scheme 


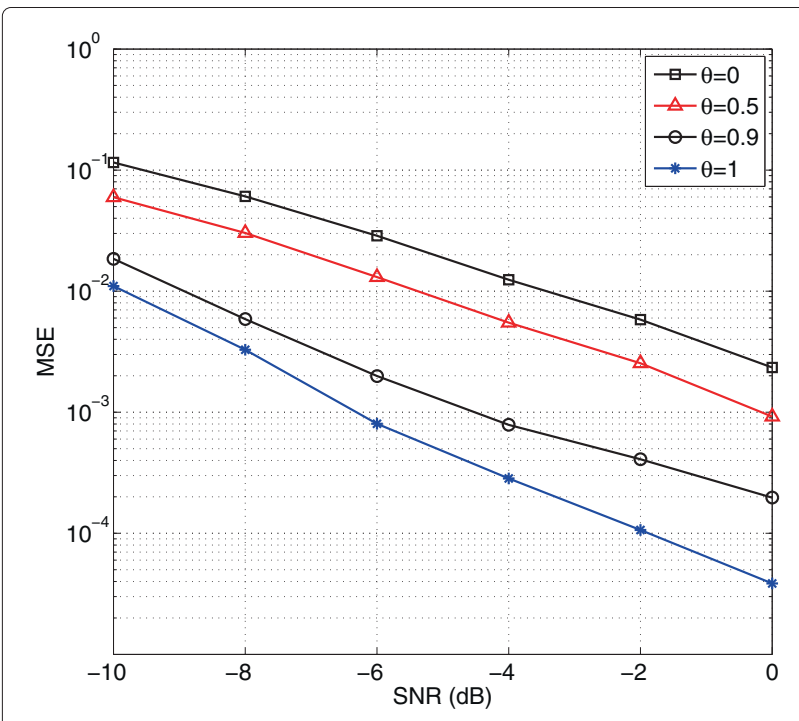

Figure 3 MSE with different values of spatial correlation coefficient, $\theta$.

was designed by exploiting the correlation between the harvested energy and vibration intensity. The structure vibration information is carried as the densities of the impulses generated by the sensors. An optimum multinode MAP detector with iterative $a$ priori probability estimation was developed to estimate the impulse densities from the spatially distributed sensor nodes. The theoretical MSE of the estimated impulse density was derived for a one-node system operating in a Rayleigh fading channel. Both the theoretical and simulation results indicated that the proposed algorithm can effectively utilize the spatial correlation among the sensors. The system can operate

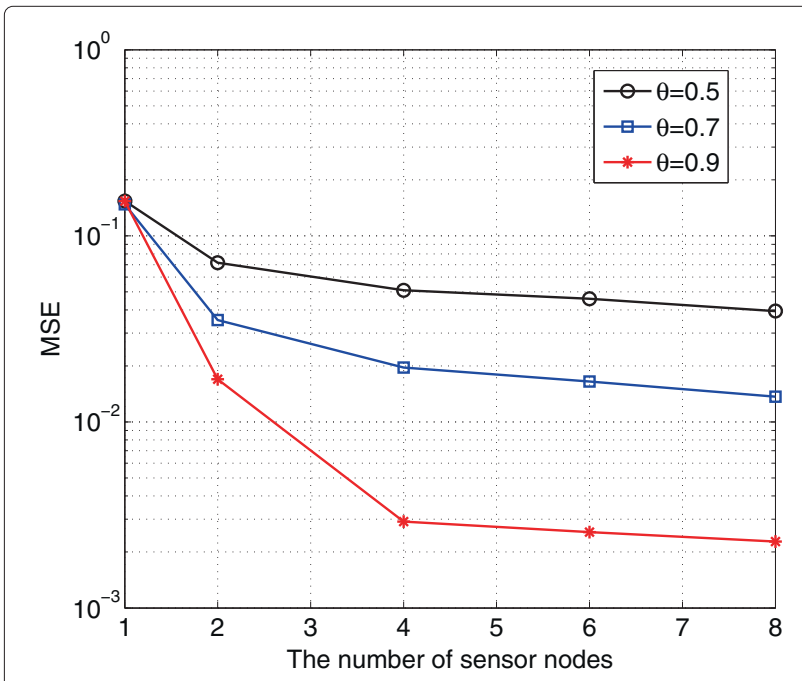

Figure 4 MSE for systems with the sub-optimum impulse density estimation. effectively at a SNR as low as $-10 \mathrm{~dB}$ without battery or external energy sources.

\section{Appendix}

\section{A. Proof of Proposition 1}

The MSE can be written as $\sigma^{2}=\frac{1}{\left(K T_{s}\right)^{2}}\left[\mathbb{E}\left(U_{n}^{2}\right)-\right.$ $\left.2 \mathbb{E}\left(U_{n} \hat{U}_{n}\right)+\mathbb{E}\left(\hat{U}_{n}^{2}\right)\right]$. Since $U_{n}$ and $\hat{U}_{n}$ are binomial RVs, we have $\mathbb{E}\left(U_{n}^{2}\right)=K p(K p-p+1)$, and $\mathbb{E}\left(\hat{U}_{n}^{2}\right)=$ $K q(N, \theta)[K q(N, \theta)-q(N, \theta)+1]$.

Based on the definition of $U_{n}$ and $\hat{U}_{n}$, we have

$$
\mathbb{E}\left(U_{n} \hat{U}_{n}\right)=\sum_{\substack{j, k=1 \\ j \neq k}}^{K} p q(N, \theta)+\sum_{k=1}^{K} \mathbb{E}\left[x_{n k} \hat{x}_{n}(k)\right] .
$$

Combining the above equations leads to (9).

\section{B. Proof of Lemma 1}

When $N=1$, the MAP detector in (5) decides $\hat{x}_{n k}=\hat{b}$ given that $x_{n k}=b$ was transmitted when

$$
\left|y_{n k}-\sqrt{E_{\mathrm{TH}}} \cdot h_{n k} \cdot \hat{b}\right|^{2}-\left|y_{n k}-\sqrt{E_{\mathrm{TH}}} \cdot h_{n k} \cdot b\right|^{2}<\eta,
$$

where $\eta=N_{0} \cdot \log \frac{P\left(x_{n k}=\hat{b}\right)}{P\left(x_{n k}=b\right)}$, and $b, \hat{b} \in \mathcal{B}$. The decision rule in (15) can be alternatively written as

$$
\mathbf{Z}<\eta-E_{\mathrm{TH}}\left|h_{n k}\right|^{2},
$$

where $\mathbf{Z}=2 \Re\left\{\sqrt{E_{\mathrm{TH}}} \cdot h_{n k} \cdot d \cdot v_{n k}\right\}, d=b-\hat{b}$. The decision variable $\mathbf{Z}$ conditioned on $h_{n k}$ and $d$ is a Gaussian random variable with 0 mean and variance $\sigma_{\mathrm{Z}}^{2}=2 N_{0} \cdot E_{\mathrm{TH}} \cdot\left|h_{n k}\right|^{2}$. Then the conditional probability

$$
\begin{aligned}
P\left\{\hat{x}_{n k}=\hat{b} \mid x_{n k}=b, h_{n k}\right\} & =P\left(\mathbf{Z}<\eta-E_{\mathrm{TH}}\left|h_{n k}\right|^{2}\right) \\
& =Q\left(\frac{E_{\mathrm{TH}}\left|h_{n k}\right|^{2}-\eta}{\sqrt{2 N_{0} \cdot E_{\mathrm{TH}} \cdot\left|h_{n k}\right|^{2}}}\right),
\end{aligned}
$$

In a Rayleigh fading channel, $f_{\left|h_{n k}\right|^{2}}(x)=\exp (-x)$, for $x \geq 0$. Then the unconditional probability is

$$
P\left\{\hat{x}_{n k}=\hat{b} \mid x_{n k}=b\right\}=\int_{0}^{\infty} Q\left(\frac{E_{\mathrm{TH}} \cdot x-\eta}{\sqrt{2 N_{0} \cdot E_{\mathrm{TH}} \cdot x}}\right) \exp (-x) d x .
$$

The results in (10) and (11) can then be obtained by substituting $(18)$ into $P\left(\hat{x}_{n k}=1\right)=p P\left(\hat{x}_{n k}=1 \mid \hat{x}_{n k}=1\right)+$ $(1-p) P\left(\hat{x}_{n k}=1 \mid \hat{x}_{n k}=0\right)$. 


\section{Competing interests}

Both authors declare that they have no competing interests.

\section{Acknowledgements}

This work was supported in part by the National Science Foundation under Grant ECCS-0917041. The authors would like to thank Dr. Shuiqing Yu for the helpful discussions on the energy harvesting properties of piezoelectric sensors. This work was supported in part by the National Science Foundation under Grants ECCS-1202075 and ECCS-1405403.

\section{Author details}

${ }^{1}$ Litepoint Corporation, 965 West Maude Avenue, Sunnyvale, CA 94085, USA.

${ }^{2}$ Department of Electrical Engineering, University of Arkansas, 3217 Bell

Engineering Center, Fayetteville, AR 72701, USA

Received: 16 October 2014 Accepted: 25 March 2015

Published online: 18 April 2015

\section{References}

1. JP Lynch, KJ Loh, A summary review of wireless sensors and sensor networks for structural health monitoring. Shock Vibration Digest. 38(2), 91-128 (2006)

2. C Escriba, J Fourniols, New real-time structural health monitoring microsystem for aircraft propeller blades. IEEE Trans. Aerospace Electronic Syst. 27, 29-41 (2012)

3. K Chintalapudi, T Fu, J Paek, N Kothari, S Rangwala, J Caffrey, R Govindan, E Johnson, S Masri. Monitoring civil structures with a wireless sensor network, in IEEE Internet Computing, vol. 10, (2006), pp. 26-34

4. S Kim, S Pakzad, D Culler, J Demmel. Health monitoring of civil infrastructures using wireless sensor networks, in Proc. 6th Intern. Sym. Information Processing in Sensor Networks. ISPN'07, (2007), pp. 254-26

5. R Cardell-Oliver, K Smettem, M Kranz, K Mayer. Field testing a wireless sensor network for reactive environmental monitoring, in Proc. IEEE Conf. Intelligent Sensors, Sensor Networks and Information Processing. SNIP'04, (2004), pp. 7-12

6. J Beutel. Fast-prototyping using the BTnode platform, in Proc. Conf. Design, Automation Test in Europe. DATE'06, (2006)

7. J Wu, N Sun. Optimal sensor density in a distortion-tolerant linear wireless sensor network, in Proc. IEEE Global Telecommun. Conf., (2010)

8. J Wu, G Zhou. A new Ultra-low power wireless sensor network with integrated energy harvesting, data sensing, and wireless communication., in Proc. IEEE Inter. Conf. on Commu., (2011)

9. S Liu, M Tomizuka, G UlsoyS, Strategic issues in sensors and smart structures. Struct. Control Health Monitor. 13, 946-957 (2006)

10. S Xu, Y Qin, C Xu, Y Wei, R Yang, Z Wang, Self-powered nanowire devices. Nat. Nanotechnol. 5, 366-373 (2010)

11. S Roundy, PK Wright, J Rabaey, A study of low level vibrations as a power source for wireless sensor nodes. J. Comput. Commu. 26, 1131-1144 (2003)

12. F Li, J Evans, S Dey, Design of distributed detection schemes for multiaccess channels. IEEE Trans. Aerospace Electronic Syst. 48, 1552-1569 (2012)

13. TM Duman, M Salehi, Decentralized detection over multiple-access channels. IEEE Trans. Aerospace Electronic Syst. 34, 469-476 (1998)

14. JJ Xiao, ZQ Luo, Universal decentralized detection in a bandwidth-constrained sensor network. IEEE Trans. Signal Process. 53, 2617-2623 (2005)

15. G Ferrari, M Martalo, R Pagliari, Decentalized detection in clustered sensor networks. IEEE Trans. Aerospace Electronic Syst. 47, 959-973 (2011)

16. G Ferrari, R Pagliari, Decentalized binary detection with noisy communication links. IEEE Trans. Aerospace Electronic Syst. 42, 1554-1562 (2008)

17. M Abramowitz, IA Stegun, Handbook of mathematical functions with formulas, graphs and mathematical tables, 9th Ed. (Dover Publications, New York, USA, 1972)

18. AD Lunn, A note on generating correlated binary variables. Biometrika. 85, 487-490 (1998)

\section{Submit your manuscript to a SpringerOpen ${ }^{\circ}$ journal and benefit from:}

- Convenient online submission

Rigorous peer review

- Immediate publication on acceptance

- Open access: articles freely available online

- High visibility within the field

- Retaining the copyright to your article

Submit your next manuscript at $>$ springeropen.com 